\title{
Blockage of PTPRJ promotes cell growth and resistance to 5-FU through activation of JAK1/STAT3 in the cervical carcinoma cell line $\mathbf{C 3 3 A}$
}

\author{
CHUN-MEI YAN ${ }^{1 *}$, YING-LING ZHAO $^{2 *}$, HONG-YI CAI ${ }^{3}$, GUO-YING MIAO ${ }^{3}$ and WEN MA ${ }^{3}$ \\ ${ }^{1}$ Lanzhou Jiao Tong University Hospital; Departments of ${ }^{2}$ Hematology, ${ }^{3}$ Radiotherapy \\ Oncology, Gansu Provincial Hospital, Lanzhou 730000, P.R. China
}

Received November 28, 2014; Accepted December 30, 2014

DOI: $10.3892 /$ or.2015.3769

\begin{abstract}
Gene therapy is a promising therapeutic approach for chemoresistant cervical cancers. Therapeutic interventions targeting the key factors contributing to the initiation and progression of cervical cancer may be a more effective treatment strategy. In the present study, we firstly determined the expression of protein tyrosine phosphatase receptor J (PTPRJ) in 8-paired human cervical tumor and non-tumor tissues. We observed a striking downregulation of PTPRJ in the human cervical tumor tissues. Next, we investigated the roles and the function mechanism of PTPRJ in the human cervical carcinoma cell line C33A by loss- and gain-of-function experiments. Our study indicated that $\mathrm{C} 33 \mathrm{~A}$ cells with loss of PTPRJ expression showed a significantly increased cell viability, rising growth and migration rate, as well as a G1-S transition. We obtained the opposite results when we overexpressed PTPRJ in C33A cells. Our further study indicated that PTPRJ levels were highly correlated with cell survival when the C33A cells were treated with 5-fluorouracil (5-FU), an important chemotherapeutic agent for cervical cancer. In addition, the signaling pathway screening assay showed an obvious alteration of the Janus kinase 1/signal transducer and activator of transcription 3 (JAK1/STAT3) pathway. PTPRJ
\end{abstract}

Correspondence to: Dr Chun-Mei Yan, Lanzhou Jiao Tong University Hospital, Lanzhou 730000, P.R. China

E-mail: yanchm123@126.com

*Contributed equally

Abbreviations: PTPRJ, protein tyrosine phosphatase receptor $\mathrm{J}$; STAT3, signal transducer and activator of transcription 3; JAK1, Janus kinase 1; Bax, B cell lymphoma 2-associated X protein; Bcl-2, B cell lymphoma 2; PI3K, phosphatidylinositol-4,5-bisphosphate 3-kinase; AKT, serine/threonine protein kinase B; HSF1, heat shock transcription factor 1; 5-FU, 5-fluorouracil; VEGF, vascular endothelial growth factor; MMP9, matrix metalloproteinase 9.

Key words: cervical cancer, proliferation, migration, apoptosis, 5 -FU negatively regulated the activation of the JAK1/STAT3 pathway by decreasing the phosphorylation levels of JAK1 and STAT3. In addition, PTPRJ also regulated the expression of the downstream factors of STAT3, such as cyclin D, Bax, VEGF and MMP2. Our results suggest that PTPRJ may be a promising gene therapy target and its therapeutic potential can be fulfilled when used alone, or in combination with other anticancer agents.

\section{Introduction}

Cervical cancer is the second most common cancer among women worldwide and it is a leading cause of cancer-related deaths (1). It is widely accepted that cervical cancer is caused by HPV infections and expression of oncogenes, such as c-MYC, Ha-RAS and ERB-2 (2,3). Cervical cancer is generally curable when detected early, but treatment of this cancer is poorly effective and is usually accompanied by adverse effects. Gene therapy is a promising therapeutic approach for chemoresistant cervical cancers (4-6). Hence, therapeutic interventions targeting the key factors contributing to the initiation and progression of cervical cancer may be a more effective treatment strategy.

The protein tyrosine phosphatase receptor $\mathrm{J}$ (PTPRJ) is a 220-kDa transmembrane protein that belongs to the family of receptor-type protein tyrosine phosphatases which are involved in multiple signaling pathways (7). PTPRJ is expressed in many cancer cell lines, including breast, colorectal, lung cancers and mammary and pancreatic tumors. Previous studies demonstrated that PTPRJ is a candidate tumor suppressor and its overexpression in different carcinoma cell lines negatively regulates cell proliferation and transformation (7-10). Mechanistically, the anti-proliferative effect of PTPRJ may account for its inhibition of growth factor signaling through the dephosphorylation of various receptor tyrosine kinases, such as FLT3, PDGFR, VEGFR2, MET, and ERK1/2 (11-15). However, the roles and function mechanism of PTPRJ in human cervical carcinoma remain largely unknown.

Here, we analyzed the expression of PTPRJ in human cervical tumor and non-tumor tissues and observed a striking downregulation of PTPRJ in cancer tissues. We further showed that PTPRJ functioned as a negative regulator of 
cell proliferation and migration in the human cervical cancer cell line C33A. In addition, sustained inhibition of PTPRJ increased the cell resistance to 5-fluorouracil (5-FU)-induced apoptosis while high levels of PTPRJ accelerated cell death. Mechanistically, PTPRJ decreased the phosphorylation levels of Janus kinase 1 (JAK1) and signal transducer and activator of signal transducer and activator of transcription 3 (stat3) and modulated the expression of the downstream factors of the JAK1/stat3 signaling pathway. The present study provided a clinical guide to the treatment of human cervical cancer.

\section{Materials and methods}

Tumor samples. For the analysis of mRNA and protein levels of PTPRJ, 8-paired human cervical tumor and non-tumor samples were obtained from patients with stage I-III adenocarcinoma and immediately frozen in liquid nitrogen and stored at the Department of Pathology, Gansu Provincial Hospital in Lanzhou, China. Immunochemistry methods were used to characterize these tissues.

Cells and reagents. Human cervical carcinoma cell line, C33A, was purchased from the American Type Culture Collection (Rockville, MD, USA) and maintained in RPMI-1640 (Invitrogen Corp., Carlsbad, CA, USA) supplemented with $10 \%$ fetal bovine serum (Hyclone, South Logan, UT, USA), $100 \mathrm{U} / \mathrm{ml}$ penicillin and $100 \mathrm{mg} / \mathrm{ml}$ streptomycin at $37^{\circ} \mathrm{C}$ in a humidified incubator with $5 \% \mathrm{CO}_{2} .5-\mathrm{FU}$ was obtained from Sigma Chemical.

Plasmid construction. PTPRJ cDNA was PCR amplified from the whole genome of HEK293T using primers for PTPRJ: 5'-ATGAAGCCGGCGGCGCGGGA-3' (sense) and 5'-TTAGGCGATGTAACCATTGG-3' (antisense). The cDNAs were ligated into pSicoR after being digested by EcoRI and XhoI to create pSicoR-PTPRJ. RNA interference vectors used in the present study were constructed inpLKO.1 puro. Target sequences are as follows: NTC (AATTCTCCGAACGTGTCACGT), shPTPRJ-1 (ACGAGTCGTCATCTAACTATA), shPTPRJ-2 (CCGATACAATGCCACCGTTTA).

Stable cell line establishment and treatment. The indicated constructs were transfected into the HEK293T cells in combination with the lentiviral packaging vectors pRSV-Rev, pMD2.G, and pCMV-VSV-G using Lipofectamine 2000 (Invitrogen). After $60 \mathrm{~h}$, the media containing the viruses were collected using a $0.22-\mu \mathrm{m}$ filter (Millipore, Bedford, MA, USA). Viral supernatant from a 6-well plate was used to infect $\sim 10^{3} \mathrm{C} 33 \mathrm{~A}$ cells for $48 \mathrm{~h}$. After a 24 -h infection, the C33A cells were selected for $48 \mathrm{~h}$ in $2.5 \mu \mathrm{g} / \mathrm{ml}$ puromycin and two colonies were chosen to expand and detect the protein levels. For 5-FU treatment, the indicated stable cell lines were plated in 6-well plates and cultured for $24 \mathrm{~h}$. Then the culture media were changed with fresh DMEM containing $20 \mu \mathrm{g} / \mathrm{ml} 5$-FU or DMSO.

Western blotting. The cells were washed with PBS and lysed in ice-cold lysis buffer consisting of $50 \mathrm{mM}$ Tris- $\mathrm{HCl}(\mathrm{pH} \mathrm{8.0)}$, $150 \mathrm{mM} \mathrm{NaCl}, 1 \% \mathrm{NP}-40,0.5 \%$ sodium deoxycholate, $0.1 \%$ SDS along with phosphatase and protease inhibitors. After sonication on ice, protein lysates were obtained by centrifugation at $12,000 \mathrm{x} \mathrm{g}$ at $4^{\circ} \mathrm{C}$ for $10 \mathrm{~min}$. The protein concentration was determined using the BCA assay. Fifty micrograms of the total protein was mixed with $4 \mathrm{X}$ loading buffer [360 mM TrisHCl (pH 6.8), 30\% glycerol and 10\% SDS]. The protein samples were then heated at $95^{\circ} \mathrm{C}$ for $5 \mathrm{~min}$ and electroseparated using $10 \%$ sodium dodecyl sulfate-polyacrylamide gel electrophoresis (SDS-PAGE) and transferred onto a PVDF membrane (Immobilon-P; Millipore). After being blocked with $0.5 \%$ skim milk, the membrane was probed with a monoclonal antibody for PTPRJ (R\&D, MAB1934), or PARP (\#9532), pro-caspase-3 (\#9665), cleaved-caspase-3 (\#9661), phosphoSTAT3 (Tyr705) (\#9145), phospho-JAK1 (Tyr1022/1023) (\#3331), STAT3 (\#9132), JAK1 (\#1013) and GAPDH (\#3683), which were purchased from Cell Signaling Technology. The membrane was then incubated with a horseradish peroxidaseconjugated secondary antibody and developed with the SuperSignal chemiluminescence kit (Immobilon Western Chemiluminescent HRP Substrate; EMD Millipore).

Cell viability and proliferation assays. C33A cells were cultured into 96-well plates at $\sim 5,000$ cells/well. After $24 \mathrm{~h}$ at $37^{\circ} \mathrm{C}$, the cells were transfected with the indicated plasmids and allowed another 48-h culture. Cell viability was assessed using the Dojindo Cell Counting Kit-8 (CCK-8; Dojindo Molecular Technologies, Kumamoto, Japan). Experiments were performed in octuplicate. For the colony formation assay, the stable C33A cells were cultured in a 6-well tissue culture plate at 500 cells/well. Cells were grown for 10-14 days. After being fixed by $4 \%$ paraformaldehyde for $30 \mathrm{~min}$, the colonies were stained with $0.1 \%$ crystal violet for $15 \mathrm{~min}$ and washed. The colonies were counted by Image $\mathbf{J}$ software.

Transwell migration assay. Stable C33A cells (200 $\mu \mathrm{l})$ in RPMI-1640 medium without FBS were loaded into the upper chambers of BD Falcon ${ }^{\mathrm{TM}}$ Cell Culture Inserts $(8-\mu \mathrm{m}$ pore size; $1 \times 10^{5}$ cells/chamber). The lower chambers were filled with $800 \mu 1$ medium (RPMI-1640 plus $10 \%$ FBS). After a $24-h$ incubation at $37^{\circ} \mathrm{C}$, the cells were fixed with paraformaldehyde and stained with 4',6-diamidino-2-phenylindole (DAPI). The stained cells on the lower side were observed under a fluorescence microscope. Three fields per chamber were photographed and the number of migrated cells was counted by Image $\mathrm{J}$ software.

Pathway screening assay. Signaling pathway arrays were conducted by a luciferase assay. Reporters HSF1-luc, phosphatidylinositol-4,5-bisphosphate 3-kinase (PI3K)/AKT-luc and STAT3-luc were purchased from Qiagen (Valencia, CA, USA). C33A cells were cotransfected with reporter plasmids and pSicoR-PTPRJ or shPTPRJ in 24-well plates. After $48 \mathrm{~h}$, the cells were lysed in $1 \mathrm{X}$ reporter lysis buffer (Promega, Madison, WI, USA) and the luciferase activity was measured by a Dual-Luciferase ${ }^{\circledR}$ Reporter assay system (Promega). Experiments were conducted in triplicates and repeated at least three times.

Real-time PCR. Total RNA was isolated using a TRIzol RNA extraction kit, and $1 \mu \mathrm{g}$ of RNA was reverse transcribed into cDNA according to the manufacturer's instructions (RevertAid 




Figure 1. Expression of PTPRJ is reduced in the human cervical tumor tissues. (A) Real-time PCR analysis of the mRNA levels of PTPRJ in 8-paired human cervical tumor (T) and non-tumor (N) tissues. (B) Western blotting of the protein levels of PTPRJ in the above patient tissues. GAPDH served as an internal control. The data represent the mean \pm SD. PTPRJ, protein tyrosine phosphatase receptor J.

First strand cDNA Synthesis kit; Thermo). Real-time PCR was performed to measure the expression of cyclin D1, Bcl-2, Bax, VEGF and MMP9. The following primers were used: GAPDH-F, 5'-TGCACCACCAACTGCTTAGC-3' and GAPDH-R, 5'-GCATGGACTGTGGTCATGAG-3'; BAX-F, 5'-GAGGATGATTGCCGCCGTGGACA-3'and BAX-R, 5'-GGTGGGGGAGGAGGCTTGAGG-3'; Bcl-2-F, 5'-ATG TGTGTGGAGAGCGTCAACC-3' and Bcl-2-R, 5'-TGAGCAG AGTCTTCAGAGACAGCC-3'; MMP9-F, 5'-CCTGGAGAC CTGAGAACCAATC-3' and MMP9-R, 5'-CCACCCGAGT GTAACCATAGC-3'; and VEGF-F, 5'-TGCCCACTGAGGA GTCCAAC-3' and VEGF-R, 5'-TGGTTCCCGAAACGC TGA G-3'. All samples were read in triplicate, and values were normalized to GAPDH expression.

Cell cycle analysis. The stable C33A cells were harvested and washed twice with PBS and fixed in 70\% cold ethanol at $4^{\circ} \mathrm{C}$ overnight. Before the analysis, the cells were washed twice with PBS, and then resuspended with $400 \mu 1 \mathrm{PBS}$ and $100 \mu \mathrm{g} / \mathrm{ml} \mathrm{RNaseA}$ (Sigma) and $50 \mu \mathrm{g} / \mathrm{ml}$ propidium iodide (PI) (Sigma). After incubation for $30 \mathrm{~min}$ at $37^{\circ} \mathrm{C}$, the cells were subjected to DNA content analysis using a FACS Calibur (Becton Dickinson, San Jose, CA, USA). The results were analyzed with Flowjo software.

Statistical analysis. SPSS 19 software was used for statistical analyses. Data are presented as means \pm SD. Statistical significance was determined by the Student's t-test in some experiments. Differences with P-values of $<0.05$ were considered significant.

\section{Results}

PTPRJ is downregulated in human cervical tumor tissues. PTPRJ is widely regarded as a tumor suppressor and its expression is decreased in various tumor types. In the present study, we analyzed the mRNA and protein levels of PTPRJ in 8-paired patient samples. We observed that both the mRNA and protein levels of PTPRJ were decreased in the majority of the examined tumor tissues $(75 \%)$. There was a $>50 \%$ decrease in 6-paired tumor and non-tumor tissues (Fig. 1A and B).

Suppression of PTPRJ promotes cell growth and migration in the cervical cancer C33A cells. To investigate the role of PTPRJ in the C33A cell line, we used construct codings for two different shRNAs against PTPRJ or a control shRNA. After puromycin selection, we obtained the stable cell lines expressing shPTPRJ. The PTPRJ shRNAs effectively decreased the mRNA and protein levels of PTPRJ, as determined by western blotting (Fig. 2A).

To assess whether the modulation of PTPRJ level influences the tumorigenetic properties of the C33A cells, we measured the cell viability, proliferation and migration capabilities by using these stable cell lines or transfected C33A cells with various doses of the indicated plasmids. By the CCK8 assay, we observed that the shPTPRJ-transfected cells showed an increased cell viability in a dose-dependent manner (Fig. 2B). By a colony formation assay, we observed that the shPTPRJinfected C33A cells exhibited increased proliferation (Fig. 2C). To further investigate the role of PTPRJ in cell migration, we performed an invasion assay by using a Transwell chamber under serum-starved conditions. We found that the shPTPRJ infection significantly enhanced the C33A cell invasive ability by $\sim 2.2$-fold relative to the scramble infection (Fig. 2D). Finally, the cell cycle distribution of the shPTPRJ/scramble-infected C33A cells was measured by use of propidium iodide staining. As shown in Fig. 2E, the percentage of shPTPRJ-infected cells in the G1 phase was considerably decreased with significantly increased percentages of cells in the $\mathrm{S}$ and G2/M phases, in comparison to the scramble-infected cells. 

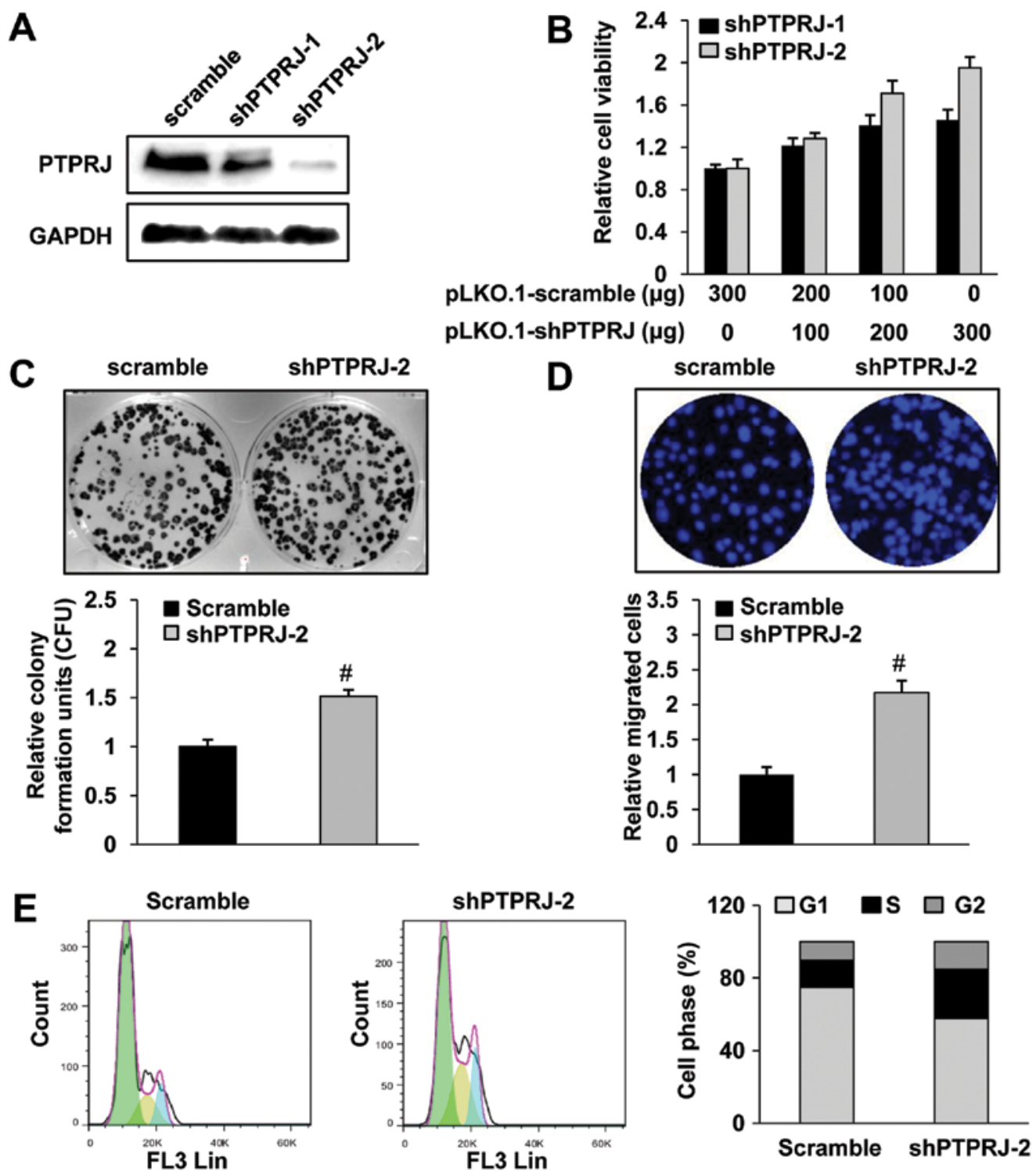

Figure 2. PTPRJ downregulation influences the cell viability, proliferation, migration and cell cycle transition. C33A cells were infected with lentivirus-scramble or lentivirus-shPTPRJ and were selected by $2 \mu \mathrm{g} / \mathrm{ml}$ puromycin to obtain stable clones. (A) Western blotting detected the protein level of PTPRJ and (B) the CCK-8 assay assessed the viability of C33A cells transfected with the indicated plasmid concentration ( ${ }^{\#} \mathrm{P}<0.05$ vs. scramble group; Student's t-test; $n=8$ ). (C) shPTPRJ promoted the proliferation of C33A cells in a colony formation assay. The lower panel indicates the quantification of the indicated colony formation units ( ${ }^{\#} \mathrm{P}<0.05$ vs. scramble group; Student's t-test; $\mathrm{n}=3$ ). (D) Transwell migration assay of scramble- or shPTPRJ-infected C33A cells. The lower panel indicates the quantification of the migrated cells ( ${ }^{\sharp} \mathrm{P}<0.05$ vs. scramble group; Student's $\mathrm{t}$-test; $\mathrm{n}=3$ ). (E) Cell cycle analysis of the indicated stable cell lines. Right panel shows the percentage of cells in the indicated cell cycle phase. GAPDH served as a loading control. The data represent the mean \pm SD. PTPRJ, protein tyrosine phosphatase receptor $\mathrm{J}$.

Re-expression of PTPRJ suppresses cell viability, proliferation and migration of the C33A cells. To identify the effects of PTPRJ re-expression on the cell proliferation of C33A cells, we generated a stable cell line with high endogenous PTPRJ levels (Fig. 3A). C33A cells with an increased PTPRJ expression had a growth disadvantage, as assessed by the CCK- 8 assay (Fig. 3B). Consistent with the previous studies in other cervical cancer cells such as HeLa (16), cell proliferation in the PTPRJ-overexpressing C33A cells was obviously reduced as compared to the corresponding control cells, confirming the antiproliferative activity of PTPRJ in the cervical cancer cell line C33A (Fig. 3C). We further used the PTPRJ-expressing C33A cells to perform a cell migration assay. Our results revealed that PTPRJ upregulation effectively reduced cell migration toward the serum starvation (Fig. 3D). By cell cycle analysis, we observed that the PTPRJ-overexpressing cells had a clearly decreased S-phase proportion and increased G2/M arrest (Fig. 3E).

Downregulation of PTPRJ enhances 5-FU-induced cell apoptosis in the C33A cells. 5-FU is an important and widely used anticancer drug, including cervical cancer. However, tumor cell resistance to 5-FU-induced cell death often occurs during clinical treatment (17). The PTPRJ agonist has been shown to induce cell apoptosis (18). To investigate the role of PTPRJ in 5-FU-induced cell apoptosis, we conducted western blotting to detect the expression of caspase- 3 and PARP, the effective markers for cell apoptosis. Our results revealed 

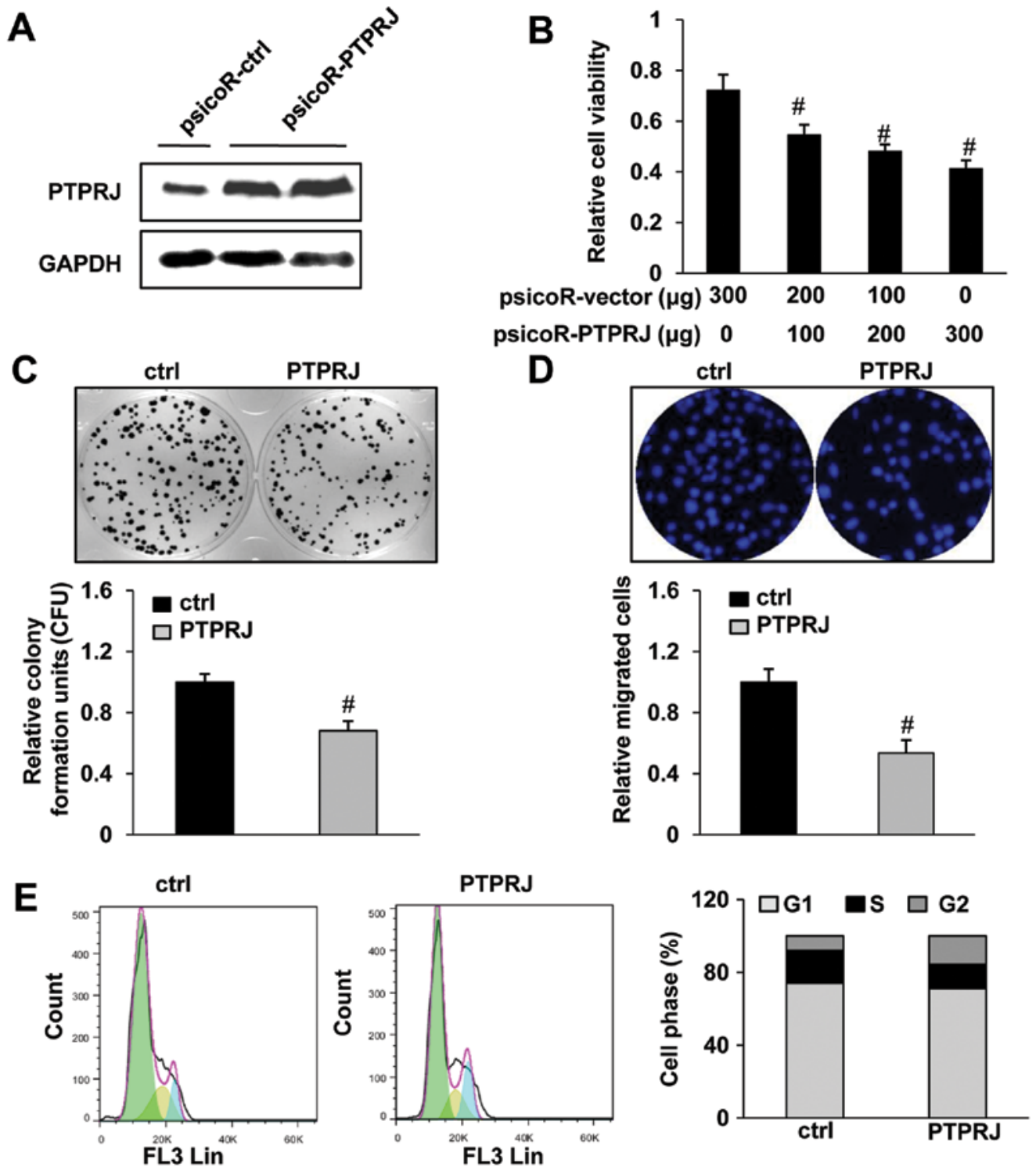

Figure 3. Ectopic overexpression of PTPRJ inhibits the tumorigenesis of C33A cells. C33A cells were infected with lentivirus-ctrl or lentivirus-pSicoR-PTPRJ and were selected by $0.5 \mathrm{mg} / \mathrm{ml} \mathrm{G418}$ to obtain stable clones. (A) Western blotting of PTPRJ levels. (B) Cell viability assay of the C33A cells transfected with the indicated plasmid concentration ( ${ }^{\sharp} \mathrm{P}<0.05$ vs. ctrl; Student's t-test; $\left.n=8\right)$. (C) Colony formation assay of the indicated stable cell lines. Lower panel shows the quantification of the indicated colony formation units ( ${ }^{\#} \mathrm{P}<0.05$ vs. scramble group; Student's $t$-test; $\left.n=3\right)$. (D) Transwell migration assay of the indicated stable cell lines. Lower panel shows the quantification of the migrated cells ( ${ }^{*} \mathrm{P}<0.05$ vs. scramble group; Student's $\mathrm{t}$-test; $\mathrm{n}=3$ ). (E) Cell cycle analysis of the indicated stable cell lines. Right panel shows the percentage of cells in the indicated cell cycle phase. GAPDH served as a loading control. The data represent the mean \pm SD. PTPRJ, protein tyrosine phosphatase receptor J.

that cleaved-caspase-3 and cleaved-PARP were upregulated after treating the wild-type C33A cells with $50 \mu \mathrm{g} / \mathrm{ml} 5$-FU, while the levels of pro-caspase-3 and full-length PARP were decreased. In the stable $\mathrm{C} 33 \mathrm{~A}$ cell line that overexpressed PTPRJ, the levels of pro-caspase-3 and full-length PARP were significantly lower, and the levels of cleaved-caspase- 3 and cleaved-PARP were higher than the levels in the control group, after treatment with an equal dose of 5-FU $(50 \mu \mathrm{g} / \mathrm{ml})$. In contrast, pro-caspase-3 and full-length PARP were increased, while cleaved-caspase- 3 and cleaved-PARP were decreased in the stable cell line expressing shPTPRJ (Fig. 4A).

Next, using the CCK-8 assay, we measured the cell viability of the wild-type C33A cells and PTPRJ/shPTPRJ stable cell lines after treatment with 5-FU. As expected, when given the 5-FU treatment, the viability of the stable cell line expressing shPTPRJ was obviously higher than the wild-type cells, while cells overexpressing PTPRJ showed a decreased cell viability, when compared with the wild-type cells (Fig. 4B).

In addition, the stable transfectants were treated with DMSO or 5-FU and subjected to FACS assays. At the basal state, the apoptotic rates of the cells transfected with the indicated genes remained unchanged. When the cells were exposed to 5-FU, ectopic overexpression of PTPRJ obviously increased the percentage of apoptotic cells, while inhibition of PTPRJ by shPTPRJ-2 attenuated the 5-FU-induced cell apoptosis, compared with the cells transfected with pSicoR-control or scramble, respectively (Fig. 4C). All things considered, the inhibition of PTPRJ decreased the sensitivity of C33A to 5-FU-induced cell apoptosis.

The activities of pJAK1 and pSTAT3 are modulated by PTPRJ in the C33A cells. PTPRJ is a receptor-like protein-tyrosine 

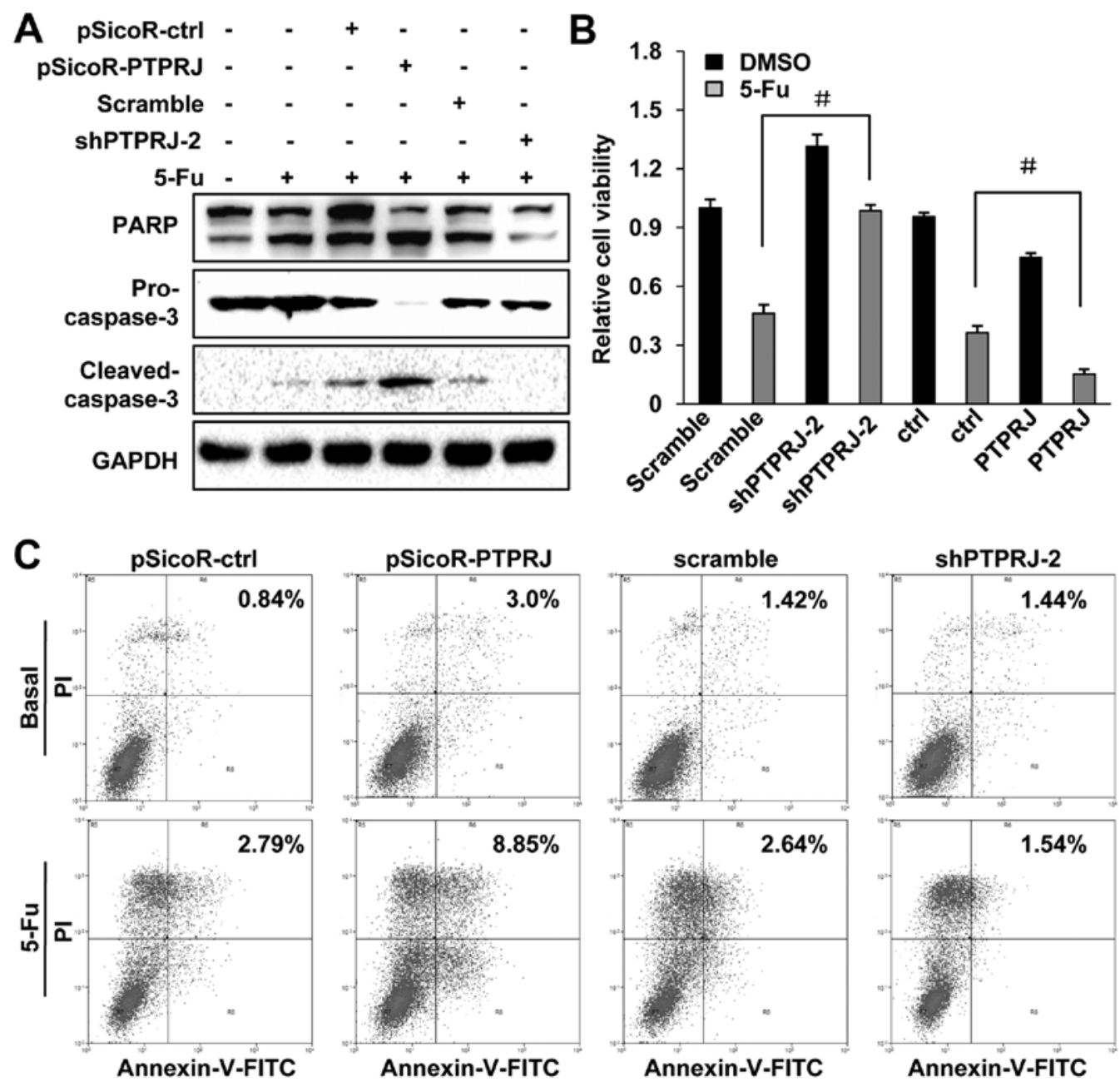

Figure 4. PTPRJ is involved in the 5-FU-induced cell apoptotic pathway. The stable C33A cells were treated with $20 \mu \mathrm{g} / \mathrm{ml} 5$-FU or DMSO. (A) Equal amounts of cell lysates were subjected to western blotting for detecting PARP, pro-caspase-3 and cleaved-caspase-3. GAPDH served as a loading control. (B) Cell viability assay of the indicated stable cell lines treated with 5 -FU or DMSO $\left({ }^{\sharp} \mathrm{P}<0.05\right.$ vs. control with DMSO treatment; Student's t-test; $\mathrm{n}=5$ ). (C) Flow cytometric analysis of cell apoptosis of the stable $\mathrm{C} 33 \mathrm{~A}$ cells transfected with the indicated plasmids. The apoptotic rates are shown at the upper right corners of the charts. The data represent the mean \pm SD. PTPRJ, protein tyrosine phosphatase receptor J; 5-FU, 5-fluorouracil.

phosphatase that directly regulates the dephosphorylation of several receptor tyrosine kinases, including FLT3, ERK, PDGFR. Omerovic et al (19) reported that PTPRJ negatively regulated serine/threonine protein kinase B (AKT) phosphorylation in Ras-mutated cancer cells. In accordance with previous studies, during the pathway screening assay, we observed that the luciferase activity of the PI3K/AKT pathway was decreased in the C33A cells transfected with PTPRJ, while inhibition of PTPRJ by shRNA led to an apparent increase in the activity of the PI3K/AKT pathway (Fig. 5A). Interestingly, we observed a similar change of heat shock transcription factor 1 (HSF1) and STAT3 pathway (Fig. 5A). STAT3 is crucial for cell proliferation, differentiation and apoptosis (20-22). STAT3 is regulated by JAK1 and other stimuli or proteins. In cervical cancer, STAT3 was reported to be correlated with clinical stage and positive-pSTAT3 patients have a favorable prognosis $(23,24)$. Given the dephosphorylation activity of PTPRJ, we analyzed the phosphorylated levels of JAK1 and STAT3. The western blotting showed that the levels of pJAK1 and pSTAT3 were decreased while the total protein levels of JAK1 and STAT3 were constant when the cells were transfected with PTPRJ. On the contrary, shPTPRJ transfection caused the upregulation of the levels of pJAK1 and pSTAT3 (Fig. 5B).

To confirm the effect of PTPRJ on the JAK1/STAT3 pathway, the mRNA levels of cyclin D1, Bax, Bcl-2, VEGF and MMP9, downstream effectors of STAT3, were measured by real-time PCR. The levels of cyclin D1, Bcl-2, VEGF and MMP9 were decreased, and the expression of Bax was increased in the cells transfected with PTPRJ (Fig. 5C). We obtained the opposite results in the cells transfected with shPTPRJ (Fig. 5D). Collectively, these results revealed a negative regulation of PTPRJ on the JAK/STAT3 signaling pathway.

\section{Discussion}

Cervical cancer is a leading cause of cancer-related deaths. PTPRJ is known to negatively regulate cell growth in many malignant tumors by phosphorylation of different substrates, but the understanding of its signaling transduction in cervical cancer is limited. The present study reports for the first time that PTPRJ plays an important role in proliferation and migration of the cervical cancer cell line C33A. PTPRJ regulates cell 
A

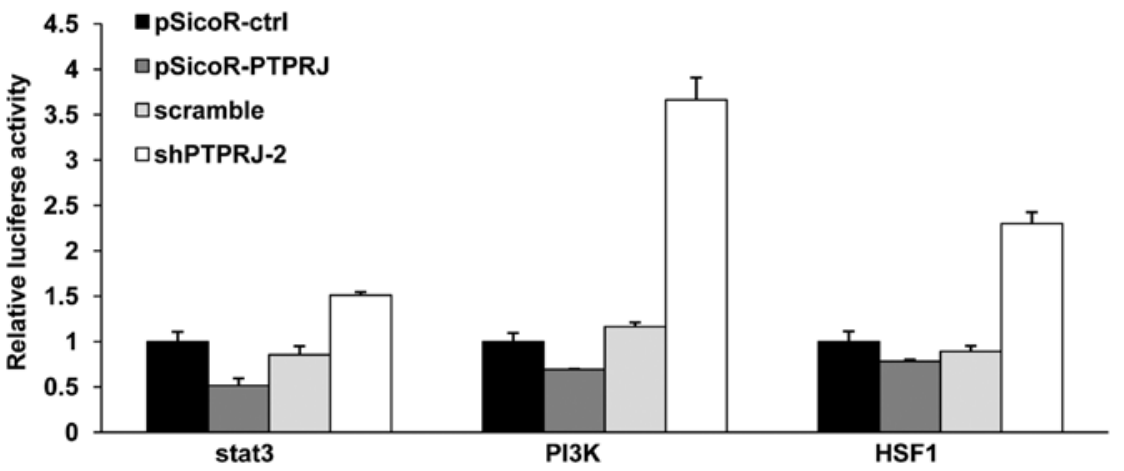

B

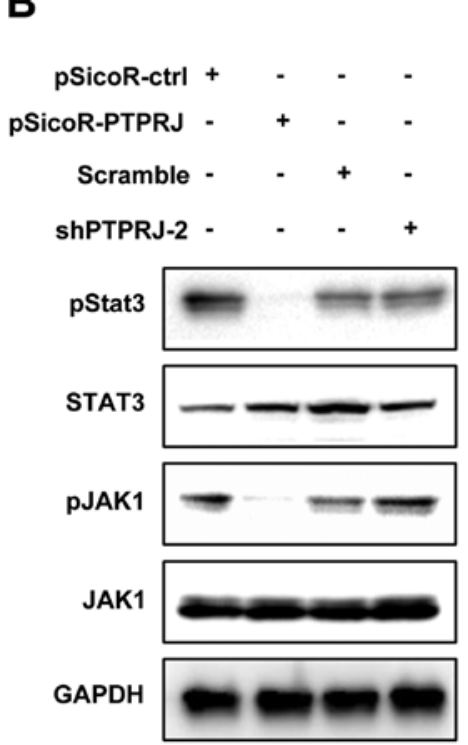

C
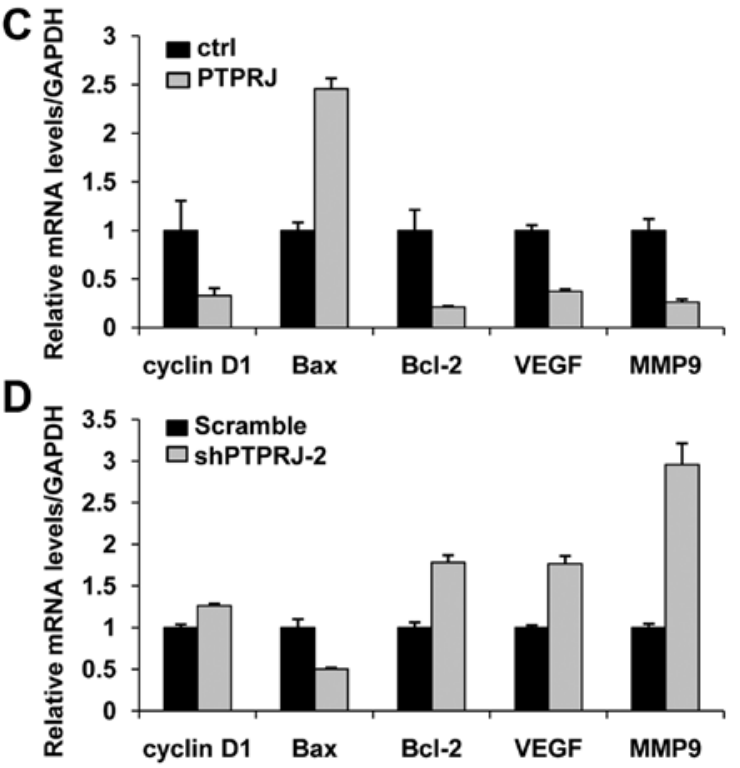

Figure 5. The activities of pJAK1 and pSTAT3 are regulated by PTPRJ. (A) The effect of PTPRJ on the activities of STAT3, PI3K and HSF1 signaling pathways was measured by luciferase reporter assay. (B) Western blotting of the levels of pJAK1, JAK1, pSTAT3 and STAT3 in C33A cells stably expressing shPTPRJ or PTPRJ. (C and D) The relative mRNA expression of STAT3 downstream genes, cyclin D1, Bcl-2, Bax, VEGF and MMP9, in C33A cells transfected with the PTPRJ or shPTPRJ-2, respectively. GAPDH served as an internal control. The data represent the mean \pm SD.

cycle progression and cell apoptosis induced by 5-FU and these processes are mediated via suppression of the phosphorylated levels of AKT and ERK. Our results suggest that PTPRJ may be a promising therapeutic target for cervical cancer treatment.

The tumor-suppressor effect of PTPRJ is well documented in breast tumors (25), liver cancer (14), acute myeloid leukemia (11) and colorectal cancer (26). Recent studies showed that the PTPRJ agonist effectively inhibits cell proliferation and triggers apoptosis of breast cancer cells (16). Accordingly, the present study showed that downregulation of PTPRJ is correlated with promotion of cell proliferation, migration and the G1-S phase transition. In contrast, the ectopic overexpression of PTPRJ effectively inhibited cancer cell growth and induced cell cycle arrest. The present study established the tumor-suppressor role of PTPRJ in C33A cells.

PTPRJ and other members of the PTP family have been reported to be involved in the cell susceptibility to a variety of human-related cancers (27). 5-FU is the most extensively studied agent in cancer treatment. However, acquired drug resistance and severe side effects limit the effectiveness of chemotherapy (17). Previous research showed that the anticancer efficacy of 5-FU can be synergistically promoted when used in combination with gene therapy, which may be tumor suppressor-based or RNAi-based (28-31). In the present study, knockdown of PTPRJ increased the C33A cell resistance to 5-FU-induced apoptosis, whereas ectopic expression of PTPRJ increased the cell apoptosis in the C33A cells.

Sufficient evidence has emerged that AKT is a key regulator of cell survival and it contributes to cell susceptibility to chemotherapy in different types of tumors $(32,33)$. The activation of AKT could be regulated by PTPRJ. Consistent with the previous study, we observed that PTPRJ significantly reduced the activity of the PI3K/AKT pathway. A novel finding of the present study was the regulatory effect of PTPRJ on the JAK1/STAT3 axis. STAT3 is a key transcription factor involved in numerous cell signal transduction networks and numerous studies have implicated the potential of STAT3 as a therapeutic target for the treatment of different types of cancer $(34,35)$. We found that PTPRJ modulated the phosphorylation levels of JAK1 and STAT3. The mRNA levels of the downstream effectors of the STAT3 pathway were altered when the cells were transfected with PTPRJ or shPTPRJ. Notably, the expression of Bcl-2, an important anti-apoptotic factor, was downregulated and the pro-apoptotic factor, Bax, was upregulated when PTPRJ was overexpressed in C33A cells. This may explain why the ectopic overexpression of PTPRJ accelerated 5-FU-induced cell apoptosis. Collectively, these results suggest that STAT3 is a substrate of PTPRJ and 
further study is needed to reveal whether this effect is due to the direct interaction between PTPRJ and STAT3.

All things considered, the present study demonstrated that blockage of PTPRJ significantly promoted tumor biological features and upregulation of PTPRJ led to inhibition of the growth of the C33A cells. Furthermore, our findings provide novel evidence that PTPRJ is a regulator of cell apoptosis induced by 5-FU. Sustained PTPRJ overexpression modulated the phosphorylated levels of JAK1/STAT3, and the downstream factors such as Bcl-2, Bax, cyclin D1, which were critical mediators of cell processes like cell apoptosis and proliferation. These data suggest that PTPRJ may serve as a potential target for cervical cancer therapy.

\section{Acknowledgements}

We thank Professor Jun Zhu and Dr Zhong Tian Bai for their assistance with the construction of the lentiviral vectors and the establishment of the stable cell lines and Yong Xiu Yang for his staff support.

\section{References}

1. Parkin DM and Bray F: Chapter 2: The burden of HPV-related cancers. Vaccine 24 (Suppl 3): S3/11-25, 2006.

2. Ronco G, Dillner J, Elfström KM, et al: Efficacy of HPV-based screening for prevention of invasive cervical cancer: follow-up of four European randomised controlled trials. Lancet 383: 524-532, 2013.

3. Pinion SB, Kennedy JH, Miller RW and MacLean AB: Oncogene expression in cervical intraepithelial neoplasia and invasive cancer of cervix. Lancet 337: 819-820, 1991.

4. Jeon YH, Lee HW, Lee YL, et al: Combined E7-dendritic cell-based immunotherapy and human sodium/iodide symporter radioiodine gene therapy with monitoring of antitumor effects by bioluminescent imaging in a mouse model of uterine cervical cancer. Cancer Biother Radiopharm 26: 671-679, 2011.

5. Koch $\mathrm{M}$ and Wiese M: Gene expression signatures of angiocidin and darapladib treatment connect to therapy options in cervical cancer. J Cancer Res Clin Oncol 139: 259-267, 2013.

6. Zheng Y, Chen H, Zeng X, et al: Surface modification of TPGSb-(PCL-ran-PGA) nanoparticles with polyethyleneimine as a co-delivery system of TRAIL and endostatin for cervical cancer gene therapy. Nanoscale Res Lett 8: 161, 2013.

7. Keane MM, Lowrey GA, Ettenberg SA, Dayton MA and Lipkowitz S: The protein tyrosine phosphatase DEP-1 is induced during differentiation and inhibits growth of breast cancer cells. Cancer Res 56: 4236-4243, 1996.

8. Iuliano R, Trapasso F, Le Pera I, et al: An adenovirus carrying the rat protein tyrosine phosphatase eta suppresses the growth of human thyroid carcinoma cell lines in vitro and in vivo. Cancer Res 63: 882-886, 2003.

9. Massa A, Barbieri F, Aiello C, et al: The expression of the phosphotyrosine phosphatase DEP-1/PTPeta dictates the responsivity of glioma cells to somatostatin inhibition of cell proliferation. J Biol Chem 279: 29004-29012, 2004.

10. Trapasso F, Iuliano R, Boccia A, et al: Rat protein tyrosine phosphatase eta suppresses the neoplastic phenotype of retrovirally transformed thyroid cells through the stabilization of p27(Kip1). Mol Cell Biol 20: 9236-9246, 2000.

11. Godfrey R, Arora D, Bauer R, et al: Cell transformation by FLT3 ITD in acute myeloid leukemia involves oxidative inactivation of the tumor suppressor protein-tyrosine phosphatase DEP-1/ PTPRJ. Blood 119: 4499-4511, 2012.

12. Kovalenko M, Denner K, Sandstrom J, et al: Site-selective dephosphorylation of the platelet-derived growth factor beta-receptor by the receptor-like protein-tyrosine phosphatase DEP-1. J Biol Chem 275: 16219-16226, 2000.

13. Lampugnani MG, Orsenigo F, Gagliani MC, Tacchetti $\mathrm{C}$ and Dejana E: Vascular endothelial cadherin controls VEGFR-2 internalization and signaling from intracellular compartments. J Cell Biol 174: 593-604, 2006.
14. Palka HL, Park M and Tonks NK: Hepatocyte growth factor receptor tyrosine kinase met is a substrate of the receptor protein-tyrosine phosphatase DEP-1. J Biol Chem 278: 5728-5735, 2003.

15. Sacco F, Tinti M, Palma A, et al: Tumor suppressor density-enhanced phosphatase-1 (DEP-1) inhibits the RAS pathway by direct dephosphorylation of ERK1/2 kinases. J Biol Chem 284: 22048-22058, 2009.

16. Ortuso F, Paduano F, Carotenuto A, et al: Discovery of PTPRJ agonist peptides that effectively inhibit in vitro cancer cell proliferation and tube formation. ACS Chem Biol 8: 1497-1506, 2013.

17. Longley DB, Harkin DP and Johnston PG: 5-fluorouracil: mechanisms of action and clinical strategies. Nat Rev Cancer 3: 330-338, 2003.

18. Paduano F, Ortuso F, Campiglia P, et al: Isolation and functional characterization of peptide agonists of PTPRJ, a tyrosine phosphatase receptor endowed with tumor suppressor activity. ACS Chem Biol 7: 1666-1676, 2012.

19. Omerovic J, Clague MJ and Prior IA: Phosphatome profiling reveals PTPN2, PTPRJ and PTEN as potent negative regulators of PKB/Akt activation in Ras-mutated cancer cells. Biochem J 426: 65-72, 2010.

20. Aggarwal BB, Kunnumakkara AB, Harikumar KB, et al: Signal transducer and activator of transcription-3, inflammation, and cancer: how intimate is the relationship? Ann NY Acad Sci 1171: 59-76, 2009.

21. Aggarwal BB, Sethi G, Ahn KS, et al: Targeting signal-transducerand-activator-of-transcription-3 for prevention and therapy of cancer: modern target but ancient solution. Ann NY Acad Sci 1091: 151-169, 2006.

22. Ihle JN: Cytokine receptor signalling. Nature 377: 591-594, 1995.

23. Sobti RC, Singh N, Hussain S, Suri V, Bharti AC and Das BC: Overexpression of STAT3 in HPV-mediated cervical cancer in a north Indian population. Mol Cell Biochem 330: 193-199, 2009.

24. Choi CH, Song SY, Kang H, et al: Prognostic significance of p-STAT3 in patients with bulky cervical carcinoma undergoing neoadjuvant chemotherapy. J Obstet Gynaecol Res 36: 304-310, 2010.

25. Smart CE, Askarian Amiri ME, Wronski A, et al: Expression and function of the protein tyrosine phosphatase receptor J (PTPRJ) in normal mammary epithelial cells and breast tumors. PLoS One 7: e40742, 2012.

26. Toland AE, Rozek LS, Presswala S, Rennert G and Gruber SB: PTPRJ haplotypes and colorectal cancer risk. Cancer Epidemiol Biomarkers Prev: 17: 2782-2785, 2008.

27. Mita Y, Yasuda Y, Sakai A, et al: Missense polymorphisms of PTPRJ and PTPN13 genes affect susceptibility to a variety of human cancers. J Cancer Res Clin Oncol 136: 249-259, 2010.

28. Lv XG, Ji MY, Dong WG, et al: EBP50 gene transfection promotes 5-fluorouracil-induced apoptosis in gastric cancer cells through Bax- and Bcl-2-triggered mitochondrial pathways. Mol Med Rep 5: 1220-1226, 2012.

29. Taiyoh H, Kubota T, Fujiwara H, et al: NK4 gene expression enhances 5-fluorouracil-induced apoptosis of murine colon cancer cells. Anticancer Res 31: 2217-2224, 2011.

30. Tian F, Fan T, Jiang Y, Zhang X and Wang X: A small interfering RNA targeting NF-kappaB p65 alone or combined with 5-FU inhibits growth of esophageal squamous cell carcinoma in nude mice. Pathol Res Pract 208: 32-38, 2012.

31. Xie Q, Liang BL, Wu YH, et al: Synergistic anticancer effect of $\mathrm{rAd} / \mathrm{P} 53$ combined with 5-fluorouracil or iodized oil in the early therapeutic response of human colon cancer in vivo. Gene 499: 303-308, 2012.

32. McCubrey JA, Steelman LS, Abrams SL, et al: Roles of the RAF/MEK/ERK and PI3K/PTEN/AKT pathways in malignant transformation and drug resistance. Adv Enzyme Regul 46: 249-279, 2006.

33. Zhang LH, Yin AA, Cheng JX, et al: TRIM24 promotes glioma progression and enhances chemoresistance through activation of the PI3K/Akt signaling pathway. Oncogene: Jan 27, 2014 (Epub ahead of prin) doi: 10.1038/onc.2013.593.

34. Kamran MZ, Patil P and Gude RP: Role of STAT3 in cancer metastasis and translational advances. Biomed Res Int 2013: 421821, 2013.

35. Gong J, Muñoz AR, Chan D, Ghosh R and Kumar AP: STAT3 down regulates LC3 to inhibit autophagy and pancreatic cancer cell growth. Oncotarget 5: 2529-2541, 2014. 